American Journal of Pharmacology and Toxicology 1 (2): 21-25, 2006

ISSN 1557-4962

(C) 2006 Science Publications

\title{
Spirulina protects against cadmium-induced hepatotoxicity in rats
}

\author{
Amr Amin ${ }^{1}$, Alaaeldin A Hamza ${ }^{1}$, Sayel Daoud ${ }^{2}$ and Waleed Hamza ${ }^{1}$ \\ ${ }^{1}$ UAE University, Al-Ain, P.O Box 17551, UAE \\ ${ }^{2}$ Pathology Department, Twam Hospital, UAE
}

\begin{abstract}
Cadmium (Cd) is an environmental and industrial cumulative pollutant that most affects many organs specially the liver. The protective effect of Spirulina, filamentous blue green algae, on $\mathrm{Cd}$ - induced oxidative stress and hepatotoxicity was evaluated in male rats. Oral administration of water extract of Spirulina for seven days followed by an acute toxic dose of Cd $\left(3.5 \mathrm{mg} \mathrm{kg} \mathrm{b.wt.}{ }^{-1}\right)$, reduced the hepatotoxicity and attenuated the Cd-induced oxidative stress. Spirulina treatment showed marked reduction in serum aminotrasferase activities and elevation in lipid peroxidation and recovery of the endogenous levels of antioxidants following Cd-intoxication. The Cd-induced hepatic histopathological changes were also minimized with the tested extract. These results suggest that Spirulina algae might play a role in reducing the toxic effect of $\mathrm{Cd}$ and its antioxidant properties seems to mediate such a protective effect.
\end{abstract}

Key words: Spirulina, cadmium, hepatic injury, protection

\section{INTRODUCTION}

Although cadmium $(\mathrm{Cd})$ is an industrial and environmental pollutant and is toxic to several tissues such as the liver, kidney and testis, the basis for its toxicity is not yet fully understood. Cadmium initially accumulates in the liver and therefore acute exposure to toxic doses of $\mathrm{Cd}$ produces apotosis and necrosis in the liver ${ }^{[1-3]}$. Overproduction of reactive oxygen species (ROS) has been considered the primary mechanism for $\mathrm{Cd}$ toxicity $^{[1,4]}$. More beneficial effects were documented for combined treatment with chelating agent and antioxidant against $\mathrm{Cd}$-induced oxidative stress in rat livers than if they were applied separately for the same purpose ${ }^{[5]}$.

Spirulina, filamentous blue-green algae (Spirulina platensis, Spirulina maxima, Spirulina fusiformis), has been consumed by man since ancient times. In Mexico and central Africa Spirulina is used as a primary food source and it is currently grown at large scale in many countries for commercial purposes ${ }^{[6]}$. Spirulina is considered as a valuable additional food source of some macro- and micronutrients including amino acids, chlorophyll, gamma-linolenic acid, carotenoids, vitamins B1 and B2 and trace elements such as iron, iodine, selenium and $z^{2 i n c}{ }^{[7]}$. Spirulina is currently recieving more attention as a nutraceutical and as a potential source of pharmaceutical targets ${ }^{[8,9]}$. Spirulina has been found to be active against several viruses including HIV (AIDS virus) and has also been reported to possess immuno-modulatory properties ${ }^{[9]}$. Anticarcinogenic and antioxidant effects have also been documented in Spirulina species. These properties were largely related to the Spirulina's phycobiliprotein phycocyanin ${ }^{[8,10]}$. In addition, Spirulina fusiformis has been shown to protect against the cisplatin- and urethane-induced genotoxicity ${ }^{[11]}$ and against mercury intoxication $^{[12]}$ in mice. Spirulina was also found to have biosorption capacity for $\mathrm{Cd}$ and lead which enhance the Spirulina's effectiveness to remove $\mathrm{Cd}$ and lead from wastewater ${ }^{[13,14]}$.

The present investigation was set to evaluate the protective effects of Spirulina species against Cdinduced acute hepatotoxicity in male rats and to study the mechanisms underlying these effects. Hepatic toxicity was assessed histopatologically and by determining serum levels of alanine and aspartate transaminases (ALT \& AST). Levels of liver antioxidants such as reduced glutathione (GSH), superoxide dismutase (SOD), catalase (CAT) and of malondialdehyde (MDA; an index of lipid peroxidation) were measured and were shown to be significantly ameliorated with spirulina.

\section{MATERIALS AND METHODS}

Chemicals: Spirulina tablets (283904-P) were purchased from DXN Marketing SDN, BHD, Malaysia). Anhydrous cadmium chloride, thiobarbituric acid, Folin's reagent, epinephrine, Superoxide dismutase, $\mathrm{H}_{2} \mathrm{O}_{2}$ and bovine albumin were purchased from Sigma Chemical Co. (St. Louis, MO, U.S.A.). All other chemicals were obtained from local commercial suppliers.

Animals: Adult male Wistar strain albino rats (150200g) were obtained from the Animal House, UAE University, U.A.E. They were maintained on standard

Corresponding Author: A. Amin, Biology Department, UAE University, Al-Ain, P.O. Box 17551, Tel: +971-3-7134381, Fax: +971-3-7671291 
pellet diet and tap water ad libitum and were kept in polycarbonate cages with wood chip bedding under a $12 \mathrm{hr}$ light/dark cycle and room temperature $22-24^{\circ} \mathrm{C}$. Rats were acclimatized to the environment for oneweek prior to experimental use. This investigation was approved by the Animal Research Ethics Committee, UAE University.

Treatment regime: Cd solution was freshly prepared in a saline solution. Spirulina was gavaged at a dose of one $\mathrm{gm} \mathrm{kg}^{-1}$ body weight and dissolved in $5 \mathrm{ml}$ distilled water. Rats were divided randomly into four groups $(n=6)$ and were treated as follows. Animals of the Cdtreated group were given a single intraperitoneal dose of Cd (3.5 mg kg b.wt. $\left.{ }^{-1}\right)$. This dose was selected as it has been previously reported to induce liver toxicity in male Wister rats ${ }^{[2]}$. Animals of the protected group were fed Spirulina solution for seven days prior Cdinjection. Then, $\mathrm{Cd}$-induced liver toxicity was initiated in these protected animals via a single dose injection of $\mathrm{Cd}$ (3.5 mg kg b.wt. $\left.{ }^{-1}\right)$. One group of rats was administered with Spirulina alone for 7 days. Animals of the control group were fed with distilled water $(5 \mathrm{ml}$ $\mathrm{kg} \mathrm{b.wt.}{ }^{-1}$ ) for 7 days and were then injected with saline (vehicle) after 10 days of water administration. Twenty four hours post $\mathrm{Cd}$ and/or vehicle injection, blood samples and liver tissues were collected and properly stored for further analyses.

Biochemical assays and histopathology: Blood was collected from the retro-orbital plexus from diethyl ether-anesthetized animals. To obtain serum, blood was collected in centrifuge tubes and centrifuged in a refrigerated centrifuge $\left(4^{\circ} \mathrm{C}\right)$ at 3000 r.p.m. for 20 minutes.

Liver tissues were dissected out and homogenized separately in ice-cold $\mathrm{KCl}(150 \mathrm{mM})$. Supernatants were collected and processed for lipid peroxidation, SOD, CAT and GSH were done. Protein was estimated by the Lowry's method as modified by Peterson ${ }^{[15]}$. The methods have been standardized in our laboratory and have been detailed elsewhere ${ }^{[16-20]}$.

For the histological examinations, pieces of liver tissues were fixed in $10 \%$ neutral phosphate-buffered formalin and hydrated tissue sections, $5 \mu \mathrm{m}$ in thickness, were stained with Hematoxylin and Eosin. The sections were examined under a Leica DMRB/E light microscope.

Statistical analysis: Data were expressed as mean \pm SE. Statistical significance between the various groups was determined using unpaired t-test and ANOVA (19).

\section{RESULTS}

Effects on liver functions: Treatment with $\mathrm{Cd}$ significantly $(\mathrm{P}<0.001)$ increased the activities of serum ALT and AST (357 and $126 \%$ respectively) compared to the control (Fig. 1).

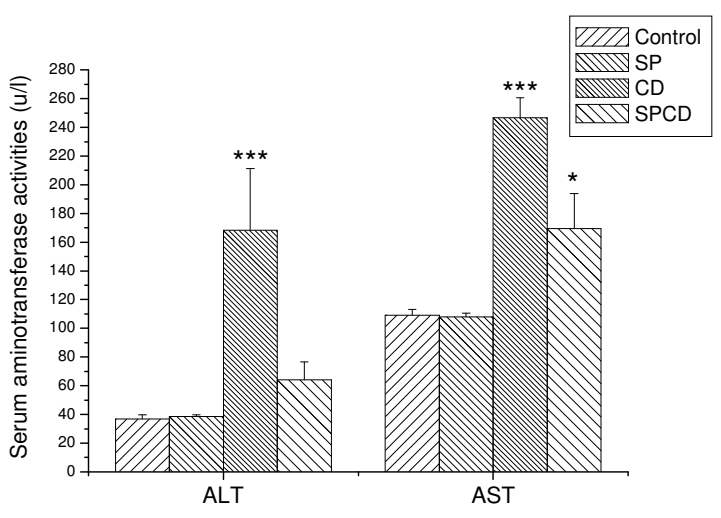

Fig. 1: Effect of SP on serum aminotransferases activities (ALT and AST) in control and CD treated rats. Columns represent the means \pm S.E.M for six animals in each group. $* \mathrm{P}<0.05$ $* * * \mathrm{P}<0.01$ vs. control
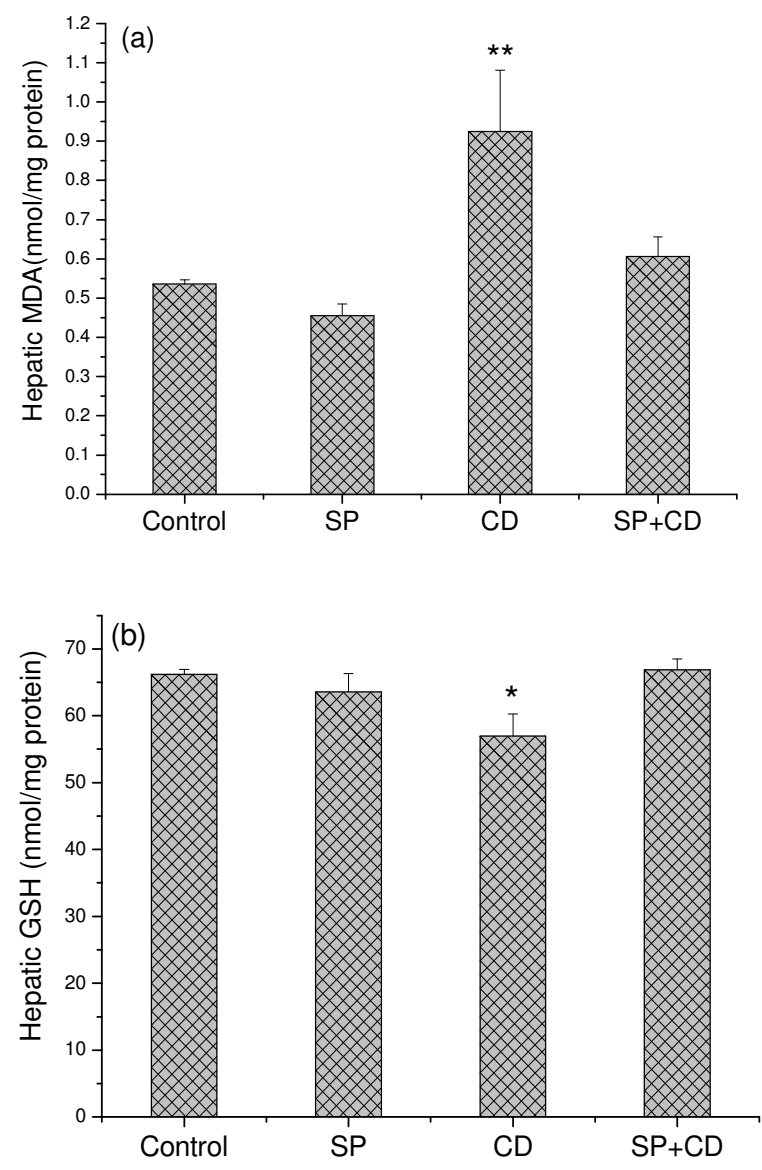

Fig. 2: Effect of SP on Hepatic (a) malondialdehyde level (MDA) and (b) reduced glutathione content $(\mathrm{GSH})$ in control and $\mathrm{CD}$ treated rats. Each column represents the mean \pm S.E.M. for six rats in each group. ${ }^{*} \mathrm{P}<0.05,{ }^{*} * \mathrm{P}<0.01$ vs. control 

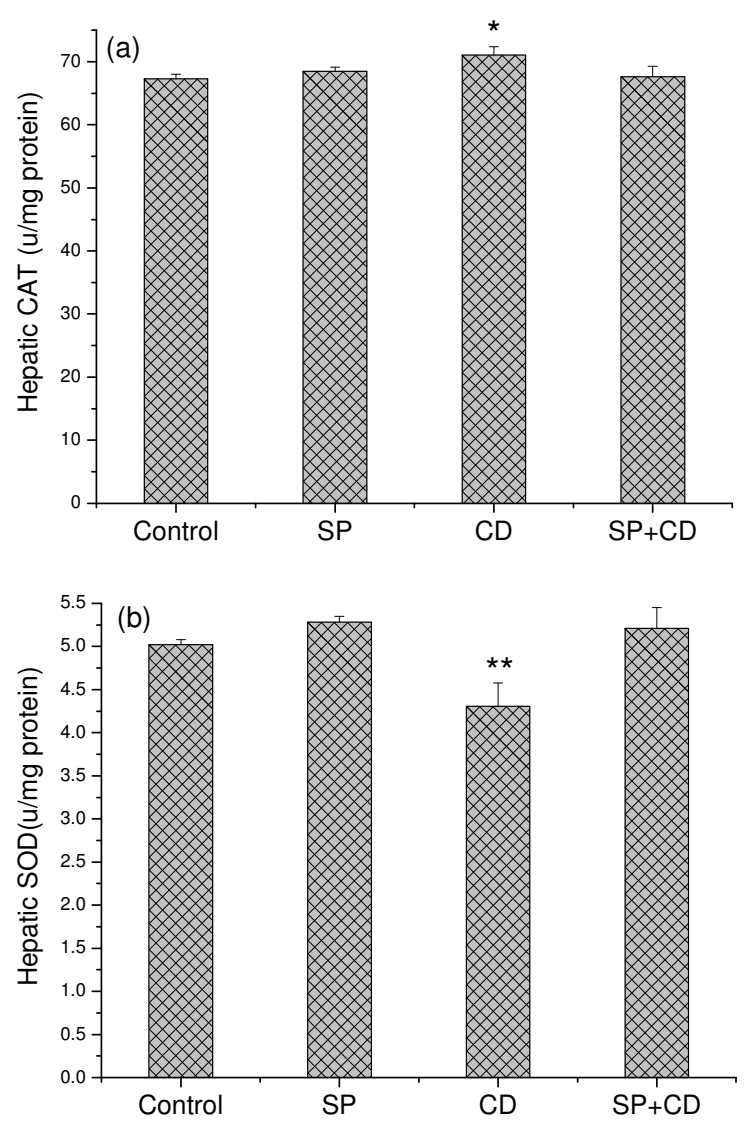

Fig. 3: Effect of SP on Hepatic on (a) catalase (CAT) \& (b) superoxide dismutase (SOD) activities (u mg ${ }^{-1}$ protein) in control and $\mathrm{CD}$ treated rats. Each column represents the mean \pm S.E.M. for six rats in each group. ${ }^{*} \mathrm{P}<0.05,{ }^{*} * \mathrm{P}<0.01$ vs. control

Administration of Spirulina alone did not show any significant changes in the serum levels of these liver enzymes. Pre-treatment with Spirulina attenuated the Cd-induced increase of serum ALT and AST activities (62 and 32\% respectively) compared to their levels in Cd-treated group (Fig. 1).

Effects on oxidative stress markers: In Cd-intoxicated rats, hepatic MDA level (Fig. 2a) was significantly $(\mathrm{P}<0.01)$ increased by about $73 \%$ compared to control group. Rats of this group have shown a significant $(\mathrm{P}<0.05)$ depletion in liver GSH level by about $14 \%$ (Fig. 2b). However, rats pre-treated with Spirulina rescued the levels of these oxidative stress markers to almost control levels.

Effects on antioxidant enzymes: A significant change in the activities of antioxidant enzymes was determined following Cd-treatment. While CAT activity was elevated $(\mathrm{P}<0.05)$, SOD activity declined $(\mathrm{P}<0.01)$ (Fig. $3)$. Levels of these oxidative stress markers were back to control values in rats pre-treated with Spirulina before Cd-intoxication.

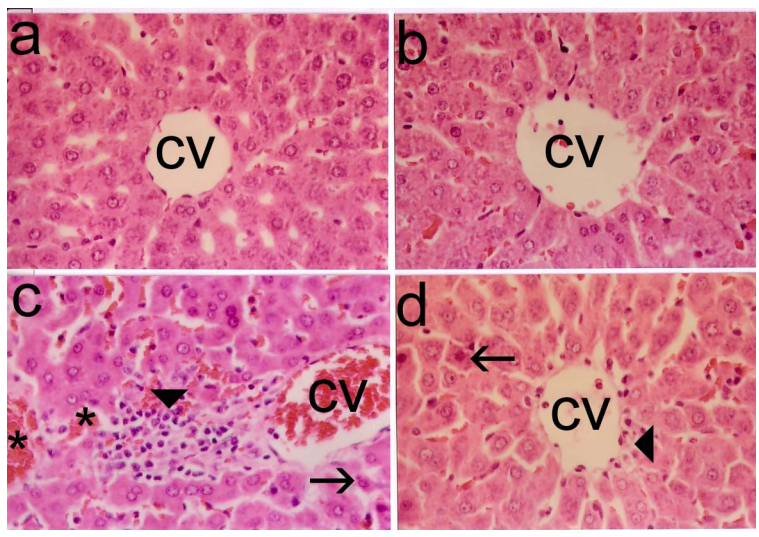

Fig. 4: Photomicrographs of liver sections in central area. (a) Liver section from control rats and from SP treated rats (b) showing the normal arrangement of hepatocytes. Liver section of CD-treated rats (c) showing extensive degeneration of hepatocytes with focal necrosis (thin arrows), vacuolated cytoplasm (thick arrows) and damaged central vein (cv). Group of rats pretreated with SP (d) before CD exhibiting relatively normal appearance of hepatocytes with moderate degeneration and focal vacuolated cells (H\&E, X 400)

Effects on histopathological changes: Hepatocytes of Cd-treated rats displayed clear cellular degeneration and loss of the distinct liver characteristic configuration. Mild necrosis was observed in all area of the lobule. The marked necrobiotic changes in the liver were mainly in the form of degenerated cells and karyolysis or pyknosis of nuclei. Histopathological changes in the liver included also congestion in central veins and sinusoids. Some hemorrhagic foci in hepatic parenchyma and infiltration of mixed inflammatory cells were observed around the necrotic hepatocytes. Interestingly, treatment with Spirulina prevented the Cd-induced necrotic lesions and the histopathological alterations. The liver appears normal except for few scattered degenerated cells (Fig. 4).

\section{DISCUSSION}

The present study evaluates the protective effect of Spirulina against liver damage induced by $\mathrm{Cd}$ in male Wister rats. Cd injection at dosage $3.5 \mathrm{mg}$ showed severe hepatic damage associated with marked increase in the serum activity of aminotransferases. These characteristic features of Cd-induced liver toxicity were similar to those previously reported by other investigators $^{[1,2]}$. Several mechanisms have been suggested for the induction of $\mathrm{Cd}$-associated hepatotoxicity ${ }^{[1]}$. In this work, Cd-treated rats showed not only a significant increase in MDA level but also a significant decrease in the hepatic levels of GSH and SOD. These results correlate well with other reports, 
where $\mathrm{Cd}$ has been shown to up regulate oxidative stress marker such as MDA and decrease the activity of antioxidants such as GSH and SOD ${ }^{[22-24]}$. Therefore, it is possible that the Cd-induced liver toxicity can be mediated by the upregulation of the reactive oxygen species (ROS; hydroxyl groups, superoxides and hydrogen peroxides) generation which in turn leads to an oxidative damage to lipid contents of membranes. Cd caused CAT activity to increase in liver. CAT is known to catalyze the removal of hydrogen peroxide and therefore its upregulation may provide a compensatory or adaptive response against elevation in hydrogen peroxide ${ }^{[17,23]}$. Over-production of ROS normally induces oxidative stress unless it was scavenged with endogenous antioxidants. Thus, overproduction of ROS could be attributed to the depletion of antioxidants or to the direct action of $\mathrm{Cd}$ on peroxidation reaction and iron-mediated peroxidation $^{[22]}$.

In the present study, administration of Spirulina alone did not significantly alter the normal level of aminotransferase enzymes, antioxidants and oxidativestress marker. Pretreatment with Spirulina prior $\mathrm{Cd}$ intoxication attenuated the levels of ALT \& AST as markers for liver function and the MDA as marker for lipid peroxidation. It also normalized the levels of both enzymatic and non-enzymatic antioxidants (GSH, SOD and CAT) in liver. This Spirulina-induced oxidative stress protection may be well correlated with previous studies, where Spirulina has been shown to restored the level of antioxidant enzymes and oxidative stress markers against cyclophosphamide and mitomycin $\mathrm{C}^{[25]}$ and cisplatin and urethane ${ }^{[11]}$ in mice. The protective effect of Spirulina against $\mathrm{Cd}$-induced oxidative stress in this study could also be attributed to its antioxidant and chelating effects. It has been reported that Spirulina possess strong antioxidant and free radical scavenging properties $^{[8,10]}$ in addition to its strong chelating ${ }^{[13,14]}$ effect. These characteristics can be attributed to the high levels of antioxidants such as vitamin B1 and B2, carotenoids and phyocyanin reported in Spirulina ${ }^{[7,8]}$.

\section{ACKNOWLEDGEMENTS}

Authors thanks A. K. Mira for proofreading this manuscript.

\section{REFERENCES}

1. Rikans, L.E. and T. Yamano, 2000. Mechanisms of cadmium-mediated acute hepatotoxicity. J. Biochem. Mol. Toxicol., 14: 110-117.

2. Tzirogiannis, K.N., G.I. Panoutsopoulos, M.D. Demonakou, R.I. Hereti, K.N. Alexandropoulou, A.C. Basayannis and M.G. Mykoniatis, 2003. Time-course of cadmium-induced acute hepatotoxicity in the rat liver: the role of apoptosis. Arch. Toxicol., 77: 694-701.
3. Takamure, Y., H. Shimada, M. Kiyozumi, A. Yasutake and Y. Imamura, 2006. A possible mechanism of resistance to cadmium toxicity in male Long-Evans rats. Environ. Toxicol. Pharmacol., 21: 231-234.

4. Casalino, E., G. Calzaretti, C. Sblano and C. Landriscina, 2002. Molecular inhibitory mechanisms of antioxidant enzymes in rat liver and kidney by cadmium. Toxicology, 179: 37-50.

5. Tandon, S.K., S. Singh, S. Prasad, K. Khandekar, V.K. Dwivedi, M. Chatterjee and N. Mathur, 2003. Reversal of cadmium induced oxidative stress by chelating agent, antioxidant, or their combination in rat. Toxicol. Lett.. 145: 211-217.

6. Chamorro, G., M. Salazar, L. Favila and H. Bourges, 1996. Pharmacology and toxicology of Spirulina alga. Rev. Invest. Clin., 48: 389-399.

7. Mazo, V. K., I.V. Gmoshinskii and I.S. Zilova, 2004. Microalgae Spirulina in human nutrition. Vopr. Pitan., 73: 45-53.

8. Pinero Estrada, J.E., P. Bermejo Bescos and A.M. Villar del Fresno, 2001. Antioxidant activity of different fractions of Spirulina platensis protean extract.

9. Khan, Z., P. Bhadouria and P.S. Bisen, 2005. Nutritional and therapeutic potential of Spirulina. Curr. Pharm. Biotecnol., 6: 373-379.

10. Wu, L.C., J.A. Ho, M.C. Shieh and I.W. Lu, 2005. Antioxidant and antiproliferative activities of Spirulina and Chlorella water extracts. J. Agric. Food Chem., 53: 4207-4212.

12. Saxena, P.S. and M. Kumar, 2004. Modulatory potential of Spirulina fusiformis on testicular phosphatases in Swiss albino mice against mercury intoxication. Indian J. Exo. Biol., 42: 998-1002.

13. Rangsayator, N., E.S. Upatham, M. Kruatrachure, P. Pokethitiyook and G. Lanza, 2002. Phytoremediation potential of Spirulina (Arthrospira) platensis: biosorption and toxicity studies of cadmium. Environ. Pollut., 119: 45-53.

11. Premkumar, K., S.K. Abraham, S.T. Santhiya and A. Ramesh, 2004. Protective effect of Spirulina fusiformis on chemical-induced genotoxicity. Fitoterapia, 75: 24-31.

14. Chen, H. and S.S. Pan, 2005. Bioremediation potential of Spirulina: toxicity and biosorption studies of lead. J. Zhejiang Univ. Sci., 6: 171-174.

15. Peterson, G.L., 1977. A simplification of the protein assay method of Lowry et al which is more generally applicable. Anol. Biochem., 83: 346 356.

16. Aebi, H., 1984. Catalase. Methods Enzymol., 105: 121-26.

17. Packer, L. and E. Cadenas, 2002. Oxidative Stress and Disease. In: Cadenas, E., Packer, L. (Ed.), Handbook Of Antioxidants. 2nd Edn. Marcel Dekker, Inc., New York, Basel, USA, pp: 5-8. 
18. Reitman, S. and S.I. Frankel, 1957. A colorimetric method for the determination of serum glutamic oxalacetic and glutamic pyruvic transaminases. Am. J. Clin. Path., 28: 56 -63.

19. Sun, M. and S. Zigman, 1978. An improved spectrophotometric assay for superoxide dismutase based on epinephrine autoxidation. Anal. Biochem., 247: 81-89.

20. Uchiyama, M. and M. Mihara, 1978. Determination of malonaldehyde precursor in tissues by thiobarbituric acid test. Anal. Biochem., 86: 271-278.

21. Van Dooran, R., C.M. Leijdekkers and P.T. Henderson, 1978. Synergistic effects of phorone on the hepatotoxicity of bromobenzene and paracetamol in mice. Toxicology, 11: 225-233.

22. Nigma, D., G.S. Shukla and A.K. Agarwal, 1999. Glutathione depletion and oxidative damage in mitochondria following exposure to cadmium in rat liver and kidney. Toxicol. Lett., 106: 151-157.
23. Pillai, A. and S. Gupta, 2005. Antioxidant enzyme activity and lipid peroxidation in liver of female rats co-exposed to lead and cadmium: effects of vitamin E and Mn $2^{+}$. Free Radic. Res., 39: 707712.

24. Chwelatiuk, E., T. Wlostowski, A. Krasowska and E. Bonda, 2006. The effect of orally administered melatonin on tissue accumulation and toxicity of cadmium in mice. J. Trace Elemen. Med. Biol., 19: 259-265.

25. Premkumar, K., A. Pachiappan, S.K. Abraham, S.T. Santhiya, P.M. Gopinath and A. Ramesh, 2001. Effect of Spirulina fusiformis on cyclophosphamide and oxidative stress in mice. Fitoterapia, 72: 906-911. 